引用格式: 韩梦瑶, 刘卫东, 唐志鹏, 等. 世界主要国家碳排放影响因素分析一一基于变系数面板模型[J]. 资源科学, 2017,39 (12) : 2420-2429. [Han M Y, Liu W D, Tang Z P, et al. Carbon emission impact factor analysis of major countries based on varying coefficient panel modeling[J]. Resources Science, 2017,39(12):2420-2429.] DOI: 10.18402/resci.2017.12.20

\title{
世界主要国家碳排放影响因素分析 基于变系数面板模型
}

\author{
韩梦瑶 $^{1,2}$, 刘卫东 ${ }^{1,2}$, 唐志鹏 ${ }^{1,2}$, 夏 炎 $^{3}$
}

(1. 中国科学院地理科学与资源研究所, 北京 100101 ;

2. 中国科学院区域可持续发展分析与模拟重点实验室, 北京 100101 ;

3. 中国科学院科技政策与管理科学研究所能源与环境政策研究中心, 北京 100190)

\begin{abstract}
摘 要: 碳减排不仅是中国政府面临的重要问题,同时是国际社会逐渐关注的热点话题。中国作为最大的温 室气体排放国, 借鉴发达国家经济发展及碳排放演变过程,有助于为自身碳减排打下坚实的基础。本文首先对比 了不同国家碳排放及碳强度的变化趋势, 然后利用变系数面板模型, 从经济发展、产业结构、城镇化率、能源结构着 手探究碳排放和碳强度的关键影响因素的作用规律, 分析中国与世界主要国家碳排放的影响因素的异同。整体来 看, 美国、日本、英国、法国、意大利、加拿大等国家均已出现不同程度的碳排放总量拐点。对比 2014 年不同国家碳 排放强度, 中国碳排放强度为 $1.24 \mathrm{~kg} /$ 美元, 远高于全球平均水平。不同影响因素对于不同国家碳排放影响程度各 不相同, 其中城市化率、可再生能源占比等对于碳排放降低均有着显著的作用。综合分析结果, 从全球的角度对比 不同国家经济增长、碳排放变化、产业结构变化、能源结构变化等规律, 对于中国应对全球气候变化、实现碳减排目 标有着重要的借鉴性意义。
\end{abstract}

关键词: 碳排放强度; 全球化; 影响因素;变系数;面板模型

DOI: $10.18402 /$ resci.2017.12.20

\section{1 引言}

气候变化已经成为影响人类可持续发展的关 键问题 ${ }^{[1]}$ 。联合国政府间气候变化专门委员会 (IPCC) 在丹麦哥本哈根发布了 IPCC 第五次评估报 告, 指出人类对气候系统的影响是明确的, 而且这 种影响在不断增强 ${ }^{[2]}$ 。作为最大的温室气体排放 国, 中国的碳减排进程一直受到世界各国的广泛关 注。为降低中国的温室气体排放, 温家宝总理在哥 本哈根世界气候变化大会上宣布, 到 2020 年, 中国 单位国内生产总值 (GDP) 的 $\mathrm{CO}_{2}$ 排放量将比 2005 年下降 40\% 45\%。而后, 中国在 2015 年“巴黎气候 峰会”上正式承诺到 2030 年实现碳排放峰值目标 ${ }^{[3]}$ 。
随着中国在全球范围内影响力逐渐扩大以及美国 新政府对巴黎协定立场的转变, 中国在全球碳减排 进程中的地位有所变化。实现上述碳减排目标不 仅成为中国政府面临的重大问题,也成为世界范围 内碳减排的核心课题, 是全社会乃至国际社会关注 的热点。

整体看,一个国家或地区碳排放的影响因素众 多,包括经济发展水平、能源结构、技术进步、管理 水平、进出口结构、减排政策等 ${ }^{[4,5]}$ 。 IPCC 报告指 出, 2000-2010年间, 人为温室气体排放量增加了 100 亿 $\mathrm{t} \mathrm{CO}_{2}$ 当量。其中, $47 \%$ 直接来自能源供应部 门, $30 \%$ 来自工业, $11 \%$ 来自交通业, $3 \%$ 来自建筑业 ${ }^{[2]}$ 。

收稿日期: 2017-09-07; 修订日期: 2017-11-14

基金项目:中华人民共和国科学技术部国家重点研发计划项目 (2016YFA0602804)。

作者简介: 韩梦瑶,女, 河北沧州人, 助理研究员, 主要研究方向为经济地理与区域发展。E-mail : hanmy@igsnrr.ac.cn

通讯作者: 刘卫东, E-mail : liuwd@igsnrr.ac.cn 
提早出现将成为自然过程,并不会明显抑制经济发

具体到产业与碳排放的关系, 交通、电力、制造业等 均被认为是碳排放增加的主要因素 ${ }^{[6-9]}$ 。根据产业 结构-能源消费、产业结构-单位能耗和能源结构-碳 排放联动模型, 张雷等发现产业结构的减排贡献高 达 $60 \%$, 能源结构的贡献也达到 $10 \%{ }^{[10]}$ 。近年来, 不 少学者对该问题进行了探讨,认为 GDP 增长、经济 结构、产业结构、能源结构均对中国碳减排有着重 要影响 ${ }^{[11,12]}$ 。由于碳排放受到能源结构、技术进步、 结构调整与减排管理等各方面的影响,相关领域的 研究受到国内外学者的广泛关注 ${ }^{[13-23]}$ 。

此外,部分研究针对碳排放强度及成本曲线展 开了细化分析 ${ }^{[24-26]}$ 。研究表明,中国在 1980-2003 年期间经历了高速的经济增长, 同时与能源相关的 碳排放呈现了明显的下降趋势 ${ }^{[27]}$ 。生产部门能源强 度的降低被认为是导致中国碳排放强度下降的主 要因素 ${ }^{[28]}$ 。而针对产业和行业碳排放强度变化的研 究表明,工业总量增长引起的能源消耗上升已经成 为导致碳排放总量迅速增长的直接原因, 并且该效 应已经抵消了能源强度效应对碳减排的贡献 ${ }^{[29]}$ 。针 对地区之间的碳转移,相关学者从国家及地区等不 同尺度开展了不同类型的细化分析 ${ }^{[19-23,30-33]}$, 并且以 此为基础对碳排放交易开展了讨论 ${ }^{[34]}$ 。也有学者指 出,由于各阶段各个因素的变化,不同发展阶段的 碳排放与经济增长的协同关系有所差异,需要进一 步细化分析 ${ }^{[35]}$ 。

另外,一些学者针对 2020 年中国碳减排路径进 行了系统分析 ${ }^{[36]}$,认为 GDP 增长、经济结构、产业结 构、能源结构均对中国碳减排有着重要影响 ${ }^{[11,12]}$ 。 同时,Cansino 等基于计量经济学方法对中国 2020 年碳排放强度进行了预测,认为 2020 年的碳排放强 度可能比 2005 年的碳排放强度降低 $50 \%$,但 2020 年 $\mathrm{CO}_{2}$ 排放总量将为 2005 年的七倍 ${ }^{[37]}$ 。另外, 刘卫 东等研究了实现中国 2020年单位 GDP 碳排放降低 $40 \% \sim 45 \%$ 目标的路径,并为《“十二五”规划纲要》的 制定提供了科学的依据 ${ }^{[17]}$ 。

尽管目前针对碳排放研究的成果层出不穷,但 具体到 2030 年中国碳排放峰值的研究为数不多 ${ }^{[38]}$ 。 现有研究成果大多基于 CGE 等定量模型展开中国 碳排放峰值的预测 ${ }^{[39-41]}$ 。值得注意的是, 不同学者 得出的结论不尽相同。部分学者指出, 碳排放峰值 展 ${ }^{[42]}$, 尤其是 Tollefson 在 Nature 上发表论文,称中国 将提前实现碳排放峰值 ${ }^{[43]}$ 。同时,也有学者指出, 为 实现 2030 年碳排放峰值,中国需进一步调整与国家 政策紧密相关的产业结构和能源结构 ${ }^{[36,44-46]}$ 。而 $\mathrm{Niu}$ 等的模拟结果显示, 中国于 2030 年前实现碳排 放达峰, 需要适当放缓经济增长速度 ${ }^{[38]}$ 。整体来看, 现有研究对中国 2030 年碳排放峰值的预测值大致 在 (92 154) 亿 $\mathrm{t}$, 且中国的人均 $\mathrm{CO}_{2}$ 排放量的峰值预

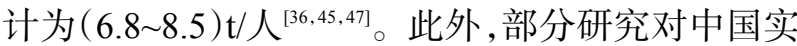
现 2030 年碳排放峰值过程中新能源和可再生能源 在一次能源供应总量中的比例进行了探究, 并量化 了碳排放强度年下降率 ${ }^{[48]}$ 。

总体来看,碳排放量分析方法主要分为两大 类。第一类是构建基于碳排放量与其影响因素的 关系模型, 如 IPAT 模型、STIRPAT模型、KAYA 恒等 式、IPAC 模型、LEAP 模型、Logistic 模型、EKC 方 法、VAR模型、3E模型、面板模型等 ${ }^{[49]}$ 。另一类是基 于历史碳排放和碳排放现状的变化趋势对未来碳 排放量进行分析，如 $\operatorname{GM}(1,1) 、 A R I M A$ 模型、神经 网络模型、回归分析。鉴于不同国家、地区的碳排 放及其决定因素的差异性,变系数模型可用于定量 分析不同国家各个影响因素的差异性和趋同性,弥 补了常参数计量经济研究的部分不足。基于中国 各省 (区市)面板数据, 相关学者已经构建了变系数 面板模型并检验了各省域碳排放的趋同性及决定 性因素 ${ }^{[50]}$ 。然而截至目前, 基于变系数面板模型从 全球角度开展系统、全面的碳排放的研究相对 较少。

随着美国对巴黎协定立场的转变, 以及中国对 全球化发展影响的变化,从宏观尺度开展碳排放及 碳强度的影响要素研究有了越发重要的意义。综 合上述背景, 本文将不同国家与中国的碳排放强 度、经济增速和碳排放量开展对比分析,引人变系 数面板数据计量经济模型,测算了世界主要国家碳 排放、经济发展、产业结构、城市化率、能源结构的 变化趋势,定量分析每个国家碳排放的差异性和趋 同性,对比不同国家各影响因素的作用效应,结合 不同阶段的碳排放及经济增长的变化趋势, 从全球 尺度为中国碳减排目标做出系统分析。 


\section{2 研究方法与数据来源}

相比时间序列模型, 面板模型能够结合数据的 时间和空间特征,使模型在估计参数时具有较大的 自由度和稳健性,在解释截面数据之间的异质性和 解决模型变量之间的多重共线上有较好的优势。 考虑到面板模型具有较好的经济揭示能力, 可对不 同时间序列的不同截面开展理论分析,本研究引人 面板模型用于检验不同国家各碳排放影响因素之 间的关联情况。

在面板分析前,本文对比了不同国家的碳排放 变化趋势、碳排放峰值、经济发展水平等,对案例国 家进行了分析。由于不同国家经济和社会的差异 化发展,经济变量的个体之间会出现异质性特征, 经典的面板模型并不适合分析类似问题,由此本文 应用固定效应变系数面板数据模型来实证分析不 同国家之间的经济水平、产业结构、城市化程度、能 源结构和碳排放的作用关系,进而分析各个国家之 间的差异性特征。基于数据稳健性的考虑,本研究 仅选取了经济发展水平较高及经济体量与中国较 相近的国家和地区,剔除了部分政权不连续的国 家。本文选取的案例国家包括美国、日本、英国、法 国、意大利、加拿大、中国、印度、澳大利亚、韩国 等。全球不同国家分产业增加值数据、人口数据和 碳排放数据分别来源于世界银行 (World Bank, http://data.worldbank.org/) ${ }^{[51]}$ 及国际能源署 (IEA, http: //www.iea.org/statistics/) ${ }^{[52]}$, 其中 GDP 为 2010 年美元不变价。本文以不同国家为研究单元, 时间 跨度为 1995-2014年组成的面板数据为研究对象。

$$
\begin{aligned}
& \text { 其一般表达式为: } \\
& y_{i t}=\alpha_{i t}+x_{i t} \beta_{i t}+\varepsilon_{i t} \\
& \quad(i=1,2, \cdots, n ; t=1,2, \cdots, T)
\end{aligned}
$$

式中 $y_{i t}$ 为被解释变量; $x_{i t}$ 为 $1 \times k$ 维解释变量 $; n$ 为 个体截面的数量、 $t$ 为时间跨度; $k$ 为解释变量的个 数;参数 $\alpha_{i t}$ 为模型的常数项或截距项; $\beta_{i t}$ 为对应于 解释变量的系数向量; $\varepsilon_{i t}$ 为随机误差项,可以发现 面板数据模型中的系数可以随着个体 $i$ 和时间 $t$ 的 不同而改变。

为了验证不同因素对各国碳排放强度的影响, 综合借鉴不同国家的实际情况,本文选取的解释变 量包括人均地区生产总值 $(G D P)$, 反映经济发展水
平; 工业占地区生产总值比重 $(I N D)$, 反映产业结构 变化; 城市人口占总人口比重 $(P O P)$, 反映城市化 水平; 新能源消费占比 $(R E W)$ 等反映能源消费 结构。

变系数模型分为固定效应变系数模型和随机 效应变系数模型。通过 Hausman 检验可对面板数 据模型进行模型设定检验。由于 Hausman 检验拒 绝了随机效应变系数模型的原假设,故而本文选取 固定效应模型开展分析。为避免模型设定的偏差， 改进参数估计的有效性,需对模型设定的合理性进 行检验。样本数据属于固定效应变截距模型还是 属于混合估计模型,可以通过 $\mathrm{F}$ 检验完成。

检验主要基于如下原假设:

$$
\begin{aligned}
& \mathrm{H} 1: b_{1}=b_{2}=\cdots=b_{N} \\
& \mathrm{H} 2: a_{1}=a_{2}=\cdots=a_{N}, b_{1}=b_{2}=\cdots=b_{N}
\end{aligned}
$$

倘若接受原假设 $\mathrm{H} 2$, 则表明样本数据属于混合 估计模型, 无需进一步检验。如果拒绝假设 H2, 则 需检验假设 $\mathrm{H} 1$; 如接受假设 $\mathrm{H} 1$, 则表明样本数据属 于固定效应变截距模型, 反之, 则认为样本数据属 于固定效应的变系数模型。

通过对不同模型的残差进行估计,具体结果 如下:

$$
\begin{aligned}
& S 1=0.164848 \quad N(T-K-1)=10 \times 15=150 \\
& S 2=0.940244 \quad N(T-1)-K=10 \times 19-4=186 \\
& S 3=4.276466 \quad N T-(K+1)=200-5=195 \\
& \text { 其中, } n=10, K=4, T=20 \\
& \text { 由此, } F 1=19.5988 \quad F(45,150) 1.32 \\
& F 2=83.3256 \quad F(36,150) 1.39
\end{aligned}
$$

通过 $\mathrm{F}$ 检验, 模型拒绝了假设 2 及假设 1 , 由此 模型采用模型 1 的形式,即为变系数面板模型。

\section{3 结果及分析}

\section{1 主要国家碳排放历史趋势}

在全球化的趋势下,中国碳减排需大力借鉴发 达国家过去和目前的经济发展实践及其能源结构 演变过程中的经验。目前,已有部分发达国家实现 碳峰值 (如果按人均量计, 则有更多国家已经实 现）。依据主要国家碳排放历史趋势,可对主要国 家碳排放类型进行划分 (见表 1)。

为分析不同国家碳排放变化趋势, 从经济增速 与碳排放强度降速的角度着手,依据表 1 中的分类， 
部分案例国家碳排放总量 (亿t)、GDP增速 $(\%)$ 、碳 排放强度下降效率 $(\%)$ 历史趋势的关系见图 1 。

\section{表 $11960-2014$ 年案例国家碳排放总量对比分析}

Table 1 Distinguish of carbon emission amounts among case countries from 1960 to 2014

\begin{tabular}{lcl}
\hline & 年均排放量 $\geqslant 6$ 亿 $\mathrm{t}$ & \multicolumn{1}{c}{ 年均排放量 $<6$ 亿 $\mathrm{t}$} \\
\hline 已出现拐点 & 美国、日本 & 英国、法国、意大利、加拿大 \\
未出现拐点 & 中国、印度 & 澳大利亚、韩国 \\
\hline
\end{tabular}

由图 1 可知,美国峰值出现在 2005 年,碳排放 总量为 57.95 亿 $\mathrm{t}$, 碳排放强度为 $0.40 \mathrm{~kg} /$ 美元, GDP 增速为 $3.35 \%$ 。日本峰值(暂时)出现在 2004 年, 碳 排放总量 12.66 亿 $\mathrm{t}$, 碳排放强度 $0.24 \mathrm{~kg}$ /美元, GDP 增速 $2.36 \%$ 。值得注意, 此处提到的峰值有可能是 阶段性的伪峰值,随着经济发展及技术变化,碳排 放有可能出现新的峰值。由图 1c 及图 1d 可知,英 国峰值出现在 1971 年,碳排放总量 6.61 亿t, 碳排放
—碳排放强度下降速率

a. 美国

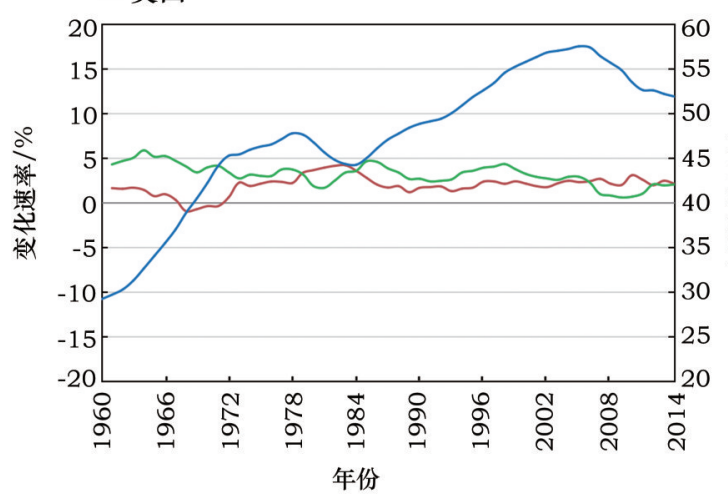

c. 英国
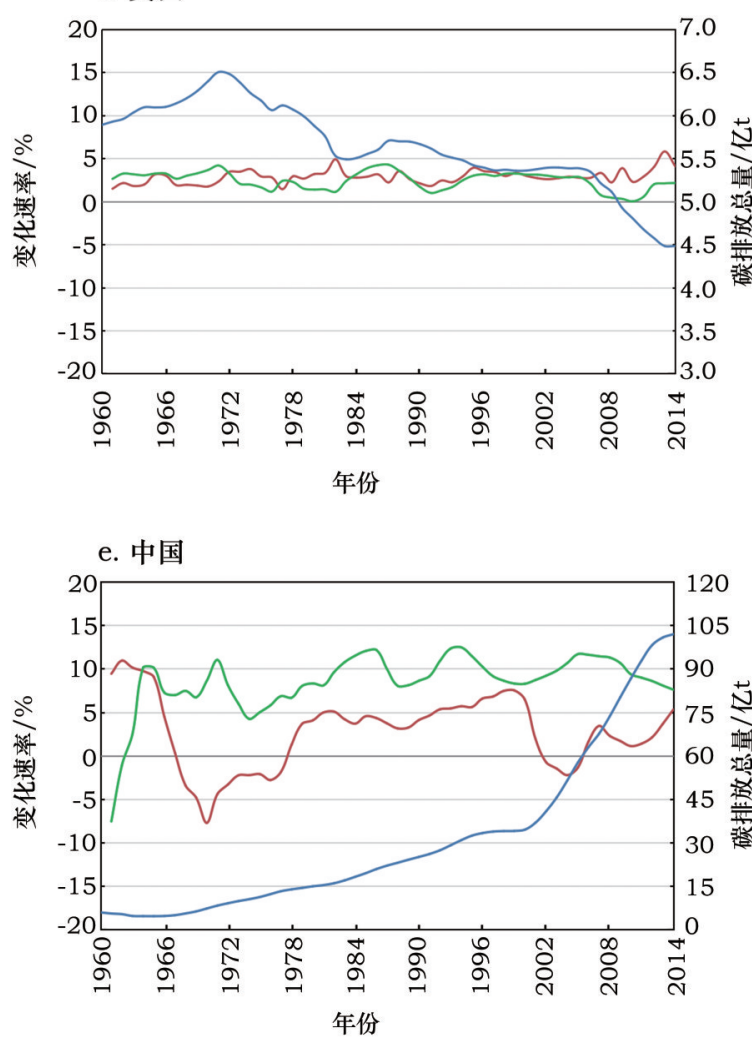

GDP增速

碳排放总量

b. 日本

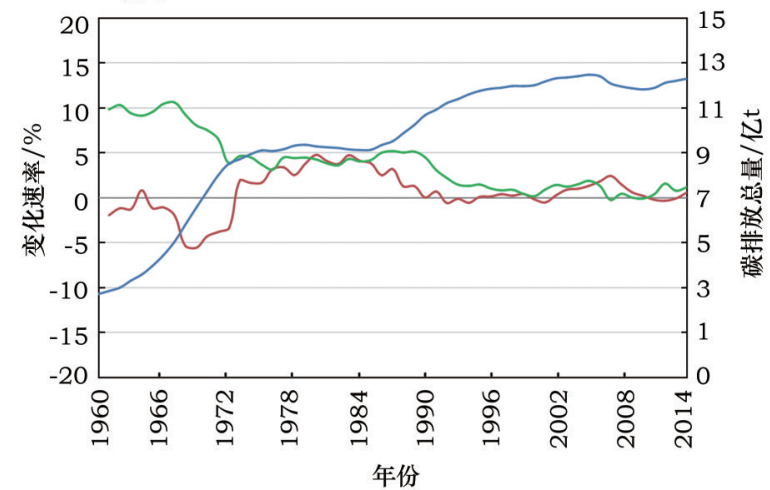

d. 法国
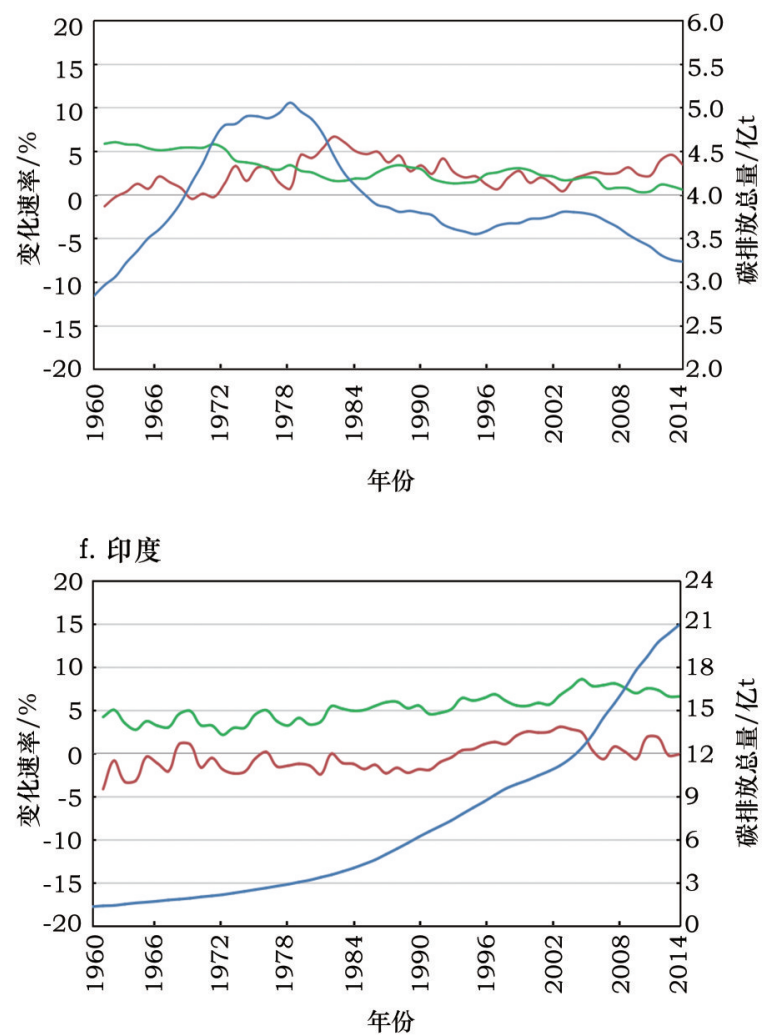

图 11960 -2014 年世界主要国家经济发展与碳排放变化关系

Figure 1 Relationship of economic development and carbon emissions in major countries from 1960 to 2014 
强度 $0.65 \mathrm{~kg}$ /美元, GDP 增速 $3.48 \%$ 。相对比,法国 峰值出现在 1979年,碳排放总量 5.29亿t, 碳排放强 度 $0.36 \mathrm{~kg}$ /美元,GDP 增速 $3.56 \%$ 。

由图 1e 及图 1f 可以看出, 截至目前, 中国和印 度均未出现碳排放峰值。整体来看, 中国碳排放强 度下降速率一直处于波动状态。1998年中国曾经 出现过碳排放总量下降的情况，当时碳排放强度下 降速度超过了 GDP 增速。2001 年后,两者差距拉 大, 中国碳排放总量迅速增长。2014年, 中国碳排 放为 102.92 亿 $\mathrm{t}$, 碳排放强度为 $1.24 \mathrm{~kg}$ /美元, 碳排放 强度下降速率为 $6.49 \%$ 。全球主要国家碳排放强度 变化情况如图 2 所示。对比不同国家碳排放强度变 化趋势, 2014 年,中国碳排放强度为 $1.24 \mathrm{~kg} /$ 美元,世 界均值约为 $0.50 \mathrm{~kg}$ /美元, OECD 为 $0.26 \mathrm{~kg} /$ 美元, 欧 盟为 $0.19 \mathrm{~kg}$ /美元, 中国碳排放强度与全球平均水平 仍有一定的差距。对照中国目前 GDP增速, 若以维 持碳峰值为前提, 可大致估算碳排放强度降速范围
为: 若 GDP增速维持在 $6.0 \%$, 碳排放强度降速至少 达到 5.6\% ; 若 GDP 增速维持在 $6.5 \%$, 碳排放强度降 速至少达到 $6.0 \%$ 。

\section{2 各国碳排放强度影响因素分析}

本文将各国家碳排放强度的影响因素进一步 进行变系数估计,得到表 2 的检验结果。基于变系 数面板模型的回归拟合较好, 拟合优度为 $99.28 \%$, 由统计量 $T$ 值及其伴随概率 $P$ 值可以看出, 不同国 家之间的差异对模型的设定有着显著影响。

参照表 1 对各个国家的分类,表 2 对不同国家 的估计结果进行了展示, 各因素对碳排放影响的具 体分析如下。GDP 是影响不同国家碳排放的显著 因素。GDP 的回归系数均为正, 说明 GDP 增加对 于碳排放有着显著的促进作用。随着经济的发展 和国内生产总值的提高, 碳排放量会随之增加。弹 性系数越大, 说明 GDP 增加会引起碳排放量的增 加。从模型解释变量的弹性系数看,除了意大利的
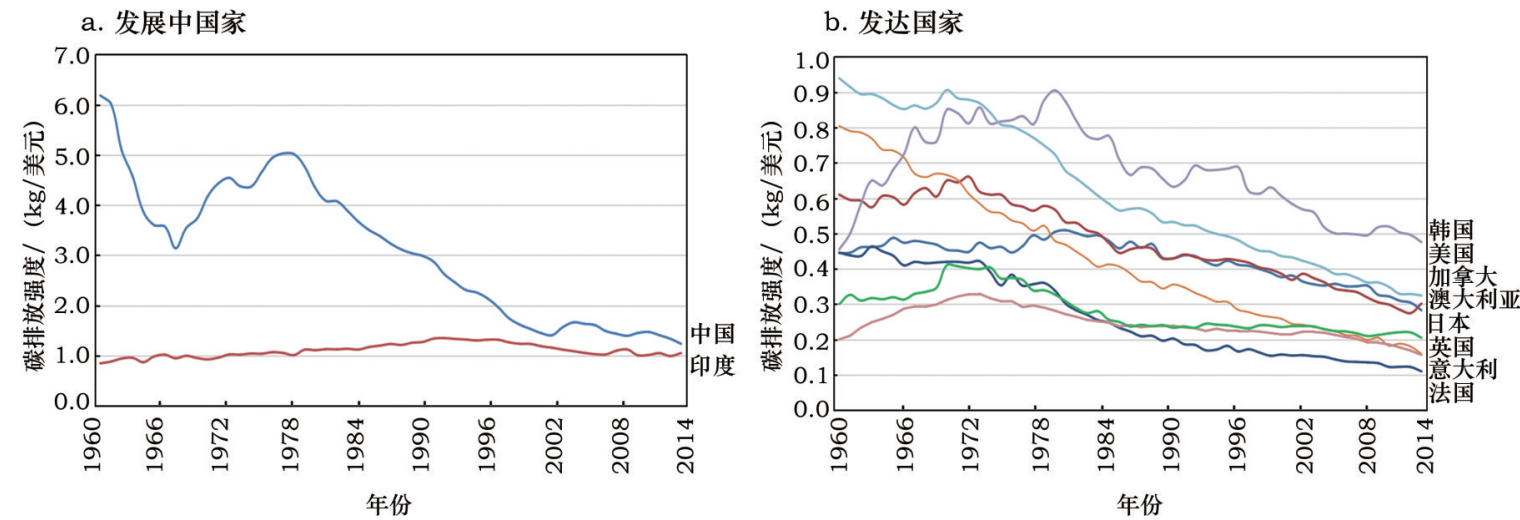

图 $21960-2014$ 年主要国家碳排放强度变化对比

Figure 2 Carbon intensity changes of main countries from 1960 to 2014

表 2 固定效应变系数面板模型的估计结果

Table 2 Estimated results of fixed-effect varying coefficient model

\begin{tabular}{|c|c|c|c|c|c|c|c|c|c|}
\hline \multicolumn{2}{|c|}{ 碳排放指标国家分类 } & \multirow{2}{*}{$\frac{G D P \text { 系数 }}{145.10}$} & \multirow{2}{*}{$\begin{array}{r}T \text { 值 } \\
6.06\end{array}$} & \multirow{2}{*}{$\begin{array}{l}I N D \text { 系数 } \\
-1.80 \mathrm{E}+04\end{array}$} & \multirow{2}{*}{$\frac{T \text { 值 }}{-0.45}$} & \multirow{2}{*}{$\frac{P O P \text { 系数 }}{-32.72 \mathrm{E}+04}$} & \multirow{2}{*}{$\frac{T \text { 值 }}{-2.37}$} & \multirow{2}{*}{$\frac{R E W \text { 系数 }}{-10.06 \mathrm{E}+04}$} & \multirow{2}{*}{$\begin{array}{c}T \text { 值 } \\
-2.77\end{array}$} \\
\hline 1 & 美国 & & & & & & & & \\
\hline & 日本 & 42.42 & 9.42 & $-1.31 \mathrm{E}+04$ & -2.64 & $-1.68 \mathrm{E}+04$ & -5.23 & $-1.08 \mathrm{E}+04$ & -0.99 \\
\hline \multirow[t]{4}{*}{2} & 法国 & 6.69 & 2.11 & $-1.73 E+04$ & -2.56 & $-3.55 E+04$ & -3.03 & $-0.58 \mathrm{E}+04$ & -1.94 \\
\hline & 英国 & 3.97 & 2.68 & $0.61 \mathrm{E}+04$ & 2.64 & $1.01 \mathrm{E}+04$ & 1.49 & $-3.02 E+04$ & -7.91 \\
\hline & 意大利 & 5.36 & 0.04 & $-14.43 \mathrm{E}+04$ & -0.67 & $-67.30 \mathrm{E}+04$ & -0.93 & $13.33 \mathrm{E}+04$ & 0.93 \\
\hline & 加拿大 & 14.47 & 2.38 & $0.74 \mathrm{E}+04$ & 2.17 & $-3.81 E+04$ & -1.70 & $-0.06 \mathrm{E}+04$ & -0.05 \\
\hline \multirow[t]{2}{*}{3} & 中国 & 2116.51 & 18.22 & $16.50 \mathrm{E}+04$ & 4.32 & $-15.61 \mathrm{E}+04$ & -4.92 & $-12.55 \mathrm{E}+04$ & -4.19 \\
\hline & 印度 & 729.40 & 2.84 & $-1.00 \mathrm{E}+04$ & -2.48 & $-1.97 \mathrm{E}+04$ & -0.54 & $-4.31 \mathrm{E}+04$ & -5.04 \\
\hline \multirow[t]{2}{*}{4} & 澳大利亚 & 13.12 & 5.11 & $0.34 \mathrm{E}+04$ & 2.95 & $-3.84 \mathrm{E}+04$ & -2.88 & $0.03 \mathrm{E}+04$ & 0.20 \\
\hline & 韩国 & 35.06 & 9.69 & $0.55 \mathrm{E}+04$ & 1.65 & $-4.98 E+04$ & -4.82 & $-1.66 \mathrm{E}+04$ & -1.17 \\
\hline
\end{tabular}


市化率变化较为敏感, 然而对于日本, 单位城市变 化率对碳排放的影响相对较小。

对于可再生能源, 除去不显著的变量, 可再生 能源占比对于不同国家的碳排放均有着下降的作 用。随着可再生能源占比的不断提升, 碳排放会随 之降低。弹性系数越大, 说明可再生能源占比的增 大会引起碳排放的相应降低。对比来看,中国和美 国的可再生能源占比提升对于碳减排有着显著的 作用。相反,法国可再生能源占比的提升对于碳减 排的影响相对较小。

综合主要国家碳排放历史趋势可以看出,不同 国家碳排放影响因素的作用效果各不相同。对比 来看, 加拿大 (阶段性)峰值出现在 2003 年, 日本峰 值出现在 2004 年, 意大利峰值出现在 2004 年, 美国 峰值出现在 2005 年, 澳大利亚峰值出现在 2009 年。通过与不同国家相对比,可以看出,各案例国 家碳排放强度的平均下降速率及影响因素趋势对 比如图 3 所示。

由图 3 可以看出, 中国碳排放强度的下降速率 一直处于波动状态, 1995 年后约为 $68.91 \%$ 。与其他 国家相对比,中国的碳排放强度降速高于英国之外 的大部分案例国家,这主要得益于中国近年来快速 增长的 GDP。对于中国来说, GDP 的增长及工业增 加值占比的增加对于碳排放增加有着重要的影响, 而城市化率及可再生能源占比对于碳排放有着一

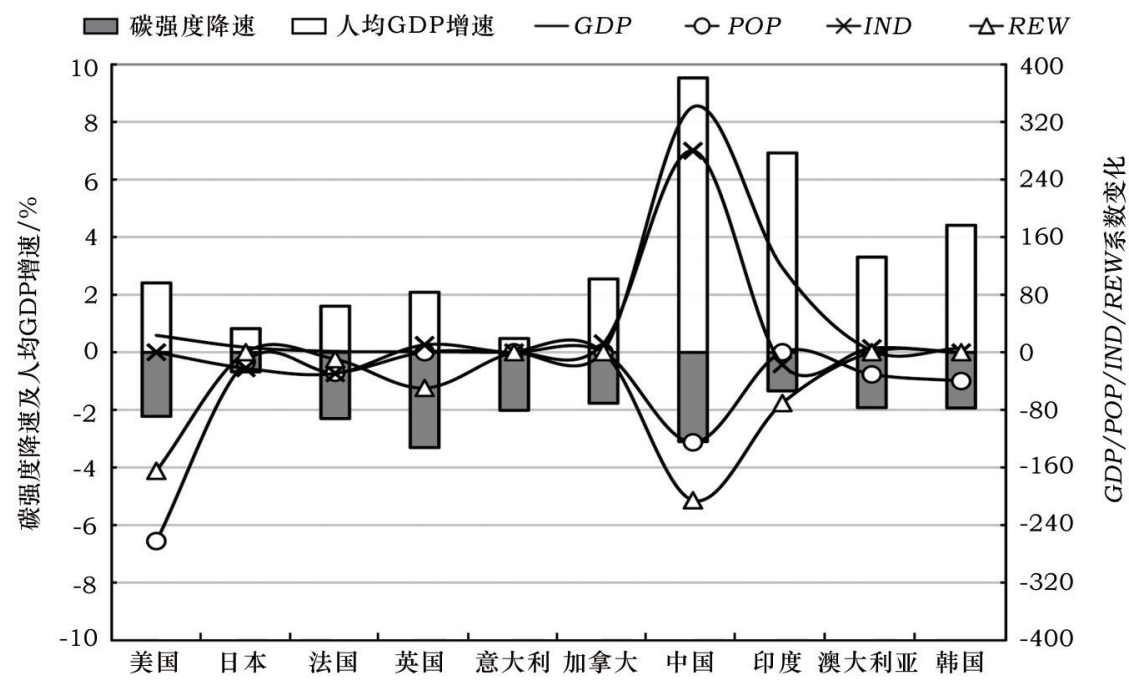

图 3 不同国家碳排放变化速率及其影响因素对比

Figure 3 Comparisons of carbon emission change rates and indicators in different countries 
定的抑制作用。对比处于不同碳排放阶段的国家， 印度的情况与中国较为类似。对于美国、澳大利亚 和韩国, 城市化率对于碳排放降低的作用较为明 显。对于英国,可再生能源占比增加对于碳排放降 低有着重要的作用。值得注意的是,经济增长对于 部分达峰国家来说没有明显的拉动作用。

从全局层面看,城市化率和可再生能源占比均 对碳排放有着重要的影响。对于发展中国家如中 国和印度,经济增长仍对碳排放有着显著的影响。 具体来看, 中国承诺到 2020 年单位国内生产总值 (GDP) 的 $\mathrm{CO}_{2}$ 排放量比 2005 年下降 $40 \%$ 45\%, 并 设定了 2030 年非化石能源占比达到 $20 \%$ 的目标。 考虑到中国长时期以煤炭为主的现状, 在未来相当 长一段时期内, 煤炭、石油、天然气等化石能源仍将 在中国能源消费结构中占据重要地位。由此,积极 调整能源结构,增加可再生能源比例有着重要的意 义。此外,碳排放量的变化同样来自于产业结构调 整以及宏观政策调控。在中国逐渐依靠市场化手 段促进节能减排的大背景下,结构调整和优化对中 国碳排放变化的影响密切。目前中国的直接碳排 放仍主要集中在工业部门,尤其是电力、钢铁、有色 金属、水泥以及建筑和交通运输等行业。通过发展 低碳产业和改造传统高碳产业, 实现从源头上降低 能耗,是中国碳减排的必经之路。

\section{4 结论及展望}

通过对比不同国家的碳排放历史变化规律,本 研究旨在分析经济发展、产业结构、城市化率、能源 结构等对碳排放的作用影响,致力于为中国碳排放 长期趋势和影响要素做出科学判断。对比不同国 家碳排放趋势,美国、日本、英国、法国、意大利、加 拿大已出现阶段性峰值。对比来看, 碳排放峰值一 般出现在 $0.50 \mathrm{~kg}$ /美元以下。具体到各影响因素, GDP 的增长及可再生能源占比增加对于中国碳排 放有着重要的影响, 而城市化率及结构调整对于中 国碳排放影响相对较小。然而,值得注意的是, 对 于美国、法国、澳大利亚、韩国等发达国家,城市化 率对于碳排放降低的作用较为明显。对于英国,可 再生能源占比对于碳排放降低有着重要的作用。

整体来看, 中国碳排放强度的下降速率一直处 于波动状态。与其他国家相对比,中国的碳排放强
度相对较高,仍有很大的下降空间。对于中国现阶 段的碳减排,调整产业结构以及提高城镇化率均有 着较为显著的作用,能源消费规模及其结构调整对 于降低总体的能源碳强度同样具有重要意义。此 外,通过发展低碳产业和改造传统高碳产业,推广 新能源技术, 实现从源头上降低碳排放是实现 2030 年碳峰值的必经之路。目前,部分发达国家已经达 到阶段性碳峰值,借鉴发达国家的经济发展及碳排 放演变过程,对比中国与主要国家碳排放变化的差 别,可以对中国 2030 年实现碳排放峰值目标起到借 鉴性意义。通过梳理世界主要国家的碳排放及经 济发展历史趋势, 从全球的角度对比不同国家经济 发展、碳排放量、产业结构、城镇化率、能源结构变 化等的规律对于中国应对全球气候变化有着借鉴 性意义。

\section{参考文献 (References) :}

[1] Aden N. The Roads to Decoupling: 21 Countries Are Reducing Carbon Emissions While Growing GDP[EB/OL]. (2016-04-05) [2017-11-13]. http://www.wri.org/blog/2016/04/roads-decoupl ing-21-countries-are-reducing-carbon-emissions-while-growing$\operatorname{gdp} /$.

[2] IPCC. Climate Change 2013: The Physical Science Basis. Contribution of Working Group I to the Fifth Assessment Report of the Intergovernmental Panel on Climate Change[M]. New York: Cambridge University Press, 2013.

[3] 中国能源研究会. 中国能源展望 2030[M]. 北京: 经济管理出版 社, 2016. [China Energy Research Society. China Energy Outlook 2030[M]. Beijing: Economy \& Management Publishing House, 2016.]

[4] 李小平, 卢现祥. 国际贸易, 污染产业转移和中国工业 $\mathrm{CO}_{2}$ 排放 [J]. 经济研究, 2010, (1): 15-26. [Li X P, Lu X X. International trade, pollution industry transfer and Chinese industries' $\mathrm{CO}_{2}$ emissions[J]. Economic Research Journal, 2010, (1): 15-26.]

[5] Zhang Y J, Wei Y M. An overview of current research on EU ETS: evidence from its operating mechanism and economic effect[J]. Applied Energy, 2010, 87(6): 1804-1814.

[6] Rehan R, Nehdi M. Carbon dioxide emissions and climate change: policy implications for the cement industry[J]. Environmental Science \& Policy, 2005, 8(2): 105-114.

[7] Schipper L, Murtishaw S, Khrushch M, et al. Carbon emissions from manufacturing energy use in 13 IEA countries: long-term trends through 1995[J]. Energy Policy, 2001, 29(9): 667-688. 
[ 8] Shrestha R M, Timilsina G R. Factors affecting $\mathrm{CO}_{2}$ intensities of power sector in Asia: a Divisia decomposition analysis[J]. Energy Economics, 1996, 18(4): 283-293.

[9] Wang W W, Zhang M, Zhou M. Using LMDI method to analyze transport sector $\mathrm{CO}_{2}$ emissions in China[J]. Energy, 2011, 36 (10): 5909-5915.

[10] 张雷, 李艳梅, 黄园淅, 等. 中国结构节能减排的潜力分析 [J]. 中国软科学, 2011, (2): 42-51. [Zhang L, Li Y M, Huang Y X, et al. Analysis on character and potential of energy saving and carbon reducing by structure evolution in China[J]. China Soft Science, 2011, (2): 42-51.]

[11] 彭斯震, 张九天. 中国 2020 年碳减排目标下若干关键经济指 标研究 [J]. 中国人口・资源与环境, 2012,22(5): 27-31. [Peng S

Z, Zhang J T. Study on key macro-economic factors and indexes in context of China's goal of carbon intensity reduction [J]. China Population, Resources and Environment, 2012, 22(5): 27 31.]

[12] Yi B W, Xu J H, Fan Y. Determining factors and diverse scenarios of $\mathrm{CO}_{2}$ emissions intensity reduction to achieve the 40 $45 \%$ target by 2020 in China: a historical and prospective analysis for the period 2005-2020[J]. Journal of Cleaner Production, 2016, 122:87-101.

[13] 曹俊文. 江西省产业部门碳排放特征及减排途径-基于 1992 2007 年投入产出分析 [J]. 经济地理, 2011, 31 (12): 2111-2115.

[Cao J W. Characteristics of Jiangxi's industrial sector carbon emissions intensity and carbon reduction approach-input-output analysis based on the 1992 to 2007[J]. Economic Geography, 2011,31(12):2111-2115.]

[14] 董军, 张旭. 中国工业部门能耗碳排放分解与低碳策略研究 [J]. 资源科学, 2010, 32 (10): 1856-1862. [Dong J, Zhang X. Decomposition of carbon emissions and low carbon strategies for industrial sector energy consumption in China[J]. Resources Science, 2010,32(10): 1856-1862.]

[15] 李方一, 刘卫东, 唐志鹏. 中国区域间隐含污染转移研究[J]. 地 理学报, 2013, 68 (6): 791-801. [Li F Y, Liu W D, Tang Z P. Study on inter-regional transfer of embodied pollution in China [J]. Acta Geographica Sinica, 2013, 68(6): 791-801.]

[16] 刘红光, 刘卫东, 唐志鹏. 中国产业能源消费碳排放结构及其 减排敏感性分析 [J]. 地理科学进展, 2010, 29(6): 670-676。 [Liu H G, Liu W D, Tang Z P. The origin source and its elasticity analysis of the $\mathrm{CO}_{2}$ emission induced by fossil fuel using industrial activities in China[J]. Progress in Geography, 2010, 29 (6) : 670-676.]

[17] 刘卫东, 张雷, 王礼茂, 等. 我国低碳经济发展框架初步研究 [J]. 地理研究, 2010,29(5): 778-788. [Liu W D, Zhang L, Wang $\mathrm{L}$ M, et al. A sketch map of low-carbon economic development in China[J]. Geographical Research, 2010, 29(5): 778-788.]
[18] 吕可文, 苗长虹, 尚文英. 工业能源消耗碳排放行业差异研究以河南省为例 $[\mathrm{J}]$. 经济地理, 2012, 32(12): 15-20. [Lv K W, Miao C H, Shang W Y. Sectoral difference in carbon emission of industrial energy consumption: a case study of Henan Province [J]. Economic Geography, 2012, 32(12): 15-20.]

[19] Feng K S, Davis S J, Li X, et al. Outsourcing $\mathrm{CO}_{2}$ within China [J]. PNAS, 2013, 110(28): 11654-11659.

[20] Liu H G, Liu W D, Fan X M, et al. Carbon emissions embodied in value added chains in China[J]. Journal of Cleaner Production, 2014, 103:362-370.

[21] Liu H G, Liu W D, Fan X M, et al. Carbon emissions embodied in demand-supply chains in China[J]. Energy Economics, 2015, 50:294-305

[22] Liu W D, Li X, Liu H, et al. Estimating inter- regional trade flows in China: a sector-specific statistical model[J]. Journal of Geographical Sciences, 2015, 25(10): 1247-1263.

[23] Liu Z, Guan D, Wei W, et al. Reduced carbon emission estimates from fossil fuel combustion and cement production in China[J]. Nature, 2015, 524(7565):335-338.

[24] 徐大丰. 碳生产率的动态变化、最终需求与中国完全碳排放 [J]. 上海经济研究, 2013, (11): 47-54. [Xu D F. The dynamic of carbon productivity, final demand and China's complete carbon emission[J]. Shanghai Journal of Economics, 2013, (11 ) : 47-54.]

[25] Carson R T. The environmental Kuznets Curve: seeking empirical regularity and theoretical structure[J]. Review of Environmental Economics and Policy, 2010, 4(1):3-23.

[26] 夏炎,范英. 基于减排成本曲线演化的碳减排策略研究 $[\mathrm{J}]$. 中 国软科学, 2012, (3): 12-22. [Xia Y, Fan Y. Study on emission reduction strategy based on evaluative $\mathrm{CO}_{2}$ abatement cost value [J]. China Soft Science, 2012, (3): 12-22.]

[27] Fan Y, Liu L C, Wu G, et al. Changes in carbon intensity in China: empirical findings from 1980-2003[J]. Ecological Economics, 2007, 62(3): 683-691.

[28] 张友国. 经济发展方式变化对中国碳排放强度的影响 [J]. 经济 研究, 2010, (4) : 120-133. [Zhang Y G. Economic development pattern change impact on China's carbon intensity[J]. Economic Research Journal, 2010, (4): 120-133.]

[29] 岳超, 胡雪洋, 贺灿飞, 等. 1995-2007 年我国省区碳排放及碳 强度的分析-碳排放与社会发展 III [J]. 北京大学学报: 自然科 学版, 2010, 46 (4) : 510-516. [Yue C, Hu X Y, He C F, et al. Provincial carbon emissions and carbon intensity in China from 1995 to 2007 (carbon emissions and social development, III) [J]. Acta Scientiarum Naturalium Universitatis Pekinensis, 2010, 46 (4) : 510-516.]

[30] 刘强, 庄幸, 姜克隽. 中国出口贸易中的载能量及碳排放量分 析 [J]. 中国工业经济, 2008, (8): 46-55. [Liu Q, Zhuang X, Jiang K J. Energy and carbon embodied in main exporting goods 
of China[J]. China Industrial Economics, 2008, (8): 46-55.]

[31] Peters G P, Hertwich E G. $\mathrm{CO}_{2}$ embodied in international trade with implications for global climate policy[J]. Environmental Science \& Technology, 2008, 42(5): 1401-1407.

[32] Xu X, Zhao T, Liu N, et al. Changes of energy-related GHG emissions in China: an empirical analysis from sectoral perspective[J]. Applied Energy, 2014, 132 (11): 298-307.

[33] Zhang B, Chen Z M, Xia X H, et al. The impact of domestic trade on China's regional energy uses: a multi- regional inputoutput modeling[J]. Energy Policy, 2013, 63: 1169-1181.

[34] Han M Y, Ji X. Alternative industrial carbon emissions benchmark based on input- output analysis[J]. Frontiers of Earth Science, 2015, 10(4): 731-739.

[35] 刘慧,成升鬼, 张雷. 人类经济活动影响碳排放的国际研究动 态 [J]. 地理科学进展, 2002, 21 (5): 420-429. [Liu H, Cheng S $\mathrm{K}$, Zhang $\mathrm{L}$. The international latest research of the impacts of human activities on carbon emissions[J]. Progress in Geography, 2002, 21(5): 420-429.]

[36] Elzen M D, Fekete H, Höhne N, et al. Greenhouse gas emissions from current and enhanced policies of China until 2030: can emissions peak before 2030? [J]. Energy Policy, 2016, 89: 224236.

[37] Cansino J M, Roma'n R, Rueda- Cantuche J M. Will China comply with its 2020 carbon intensity commitment?[J]. Environmental Science \& Policy, 2015, 47: 108-117.

[38] Niu S, Liu Y, Ding Y, et al. China's energy systems transformation and emissions peak[J]. Renewable and Sustainable Energy Reviews, 2016, 58: 782-795.

[39] 林伯强, 李江龙. 环境治理约束下的中国能源结构转变-基于 煤炭和 CO2 峰值的分析 [J]. 中国社会科学, 2015, (9): 84-107. [Lin P Q, Li J L. Transformation of China's energy structure under environmental governance constraints: a peak value analysis of coal and carbon dioxide[J]. Social Sciences in China, 2015, (9): 84-107.]

[40] 林伯强, 孙传旺. 如何在保障中国经济增长前提下完成碳减排 目标 [J]. 中国社会科学, 2011, (1): 64-76. [Lin B Q, Sun C W How can China achieve its carbon emission reduction target while sustaining economic growth?[J]. Social Sciences in China, 2011, (1): 64-76.]

[41] 杨朝, 刘纪显, 吴兴弯. 基于 DSGE 模型的碳减排目标和碳排 放政策效应研究[J]. 资源科学, 2014,36 (7): 1452-1461. [Yang
A, Liu J X, Wu X Y. Carbon reduction targets and effects of emissions policy based on DSGE modeling $[\mathrm{J}]$. Resources Science, 2014, 36(7): 1452-1461.]

[42] Zhang X L, Karplus V J, Qi T Y, et al. Carbon emissions in China: how far can new efforts bend the curve?[J]. Energy Economics, 2016, 54: 388-395.

[43] Tollefson J. China's carbon emissions could peak sooner than forecast[J]. Nature, 2016, 531(7595): 425-426.

[44] 程路, 邢璐. 2030年碳排放达到峰值对电力发展的要求及影响 分析 [J]. 中国电力, 2016, 49(1): 174-177. [Cheng L, Xing L. Analysis of requirement and impact of power development under the peak carbon emissions in 2030[J]. Electric Power, 2016,49(1):174-177.]

[45] 柴麒敏,徐华清. 基于 IAMC 模型的中国碳排放峰值目标实现 路径 [J]. 中国人口・资源与环境, 2015, 25(6): 37-46. [Chai Q $\mathrm{M}, \mathrm{Xu} \mathrm{H}$ Q. Modeling carbon emission peaking pathways in China based on integrated assessment model IAMC[J]. China Population, Resources and Environment, 2015, 25(6):37-46.]

[46] Chai Q M, Xu H Q. Modeling an emissions peak in China around 2030: Synergies or trade- offs between economy, energy and climate security[J]. Advances in Climate Change Research, 2014, 5(4): 169-180.

[47] Yuan J H, Xu Y, Hu Z, et al. Peak energy consumption and $\mathrm{CO}_{2}$ emissions in China[J]. Energy Policy, 2014, 68(2): 508-523.

[48] He J K. An analysis of China's $\mathrm{CO}_{2}$ emission peaking target and pathways[J]. Advances in Climate Change Research, 2014, 5 (4): 155-161

[49] York R, Rosa E A, Dietz T. STIRPAT, IPAT and ImPACT: analytic tools for unpacking the driving forces of environmental impacts[J]. Ecological Economics, 2003, 46(3):351-365.

[50] 吴玉鸣. 中国省域碳排放异质性趋同及其决定因素研究-基于 变参数面板数据计量经济模型的实证 $[\mathrm{J}]$. 商业经济与管理, 2015, (8) : 66- 74. [Wu Y M. Study on heterogeneous convergence and determinants of carbon dioxide emissions: based on positive study of varying parameter panel data model[J]. Journal of Business Economics, 2015, (8): 66-74.]

[51] International Energy Agency. IEA Energy Atlas[EB/OL]. (201710-01)[2017-11-13]. http://www.iea.org/statistics/.

[52] World Bank. Featured Indicators[EB/OL]. (2017-10-30) [201711-13]. https://data.worldbank.org/indicator/. 


\title{
Carbon emission impact factor analysis of major countries based on varying coefficient panel modeling
}

\author{
HAN Mengyao ${ }^{1,2}$, LIU Weidong ${ }^{1,2}$, TANG Zhipeng ${ }^{1,2}$, XIA Yan $^{3}$ \\ (1. Institute of Geographic Sciences and Natural Resources Research, Chinese Academy of Sciences, Beijing 100101, China; \\ 2. Key Laboratory of Regional Sustainable Development Modeling, Chinese Academy of Sciences, Beijing 100101, China; \\ 3. Center for Energy and Environmental Policy research, Institute of Policy and Management, Chinese Academy of Sciences, Beijing 100190, China)
}

\begin{abstract}
China's carbon emission reduction is an essential issue for the Chinese government and also of international concern. Since China has become the largest carbon emitter, analyzing the evolution of economic development and carbon emissions of developed countries as well as carrying out detailed analyses on China's carbon emission and carbon intensity changes will be an essential reference for China's carbon reduction process. Through detailed analysis of trends in carbon emissions and carbon intensities in different countries, we applied the variable coefficient panel model to assess key factors including the industrial structure, energy structure, urbanization rate and economic development. Based on the above-mentioned results, we compared differences in carbon emissions and carbon intensities between China and major countries in the world with different influence of basic impact factors taken into consideration. Overall, the United States, Japan, United Kingdom, France, Italy and Canada have reached a carbon emission peak to varying degrees. In contrast to carbon intensities in different countries, China's intensity is quantified as $1.24 \mathrm{~kg} / \mathrm{USD}$, which is still much higher than the global average. Overall, different factors have different degrees of influence on countries, but the urbanization rate and renewable energy ratio contribute significantly to carbon emission reduction when compared with others. Comparing the economic growth, carbon emissions, industrial structure and energy structure of different countries will lay a solid foundation for China and provide an essential support for the Chinese government to deal with global climate change and achieve carbon emission reduction targets at a macro scale.
\end{abstract} Key words : carbon intensity; globalization; impact factor; variable coefficient; panel model 